\title{
Geometric integration of a wave-vortex model
}

\author{
C. J. Cotter ${ }^{*}$ and S. Reich \\ Department of Mathematics, Imperial College of Science, Technology and \\ Medicine, 180 Queen's Gate, London, SW7 2AZ
}

\begin{abstract}
We introduce a Hamiltonian particle-mesh method for two-dimensional advection under incompressible flow fields. The method is applied to a simplified shallow-water model, called the weak-wave model, which combines slow nonlinear vortical motion with fast linear wave propagation. The advantages of the conservative particle-mesh method are demonstrated by means of the adiabatic energy exchange between vortical and wave motion. More generally, the proposed method is applicable to stable and efficient long-time simulations of other simplified geophysical fluid models.
\end{abstract}

Key words: geometric integration, symplectic integrators, geophysical fluid dynamics, balance, exponential estimates

\section{Introduction}

The paper is motivated by the need for stable and efficient long-time integration methods for simplified atmospheric fluid models such as the quasigeostrophic equations [12] and the weak-wave model of NorE \& SHEPHERD [9]. The goal is to compute time series and statistical ensembles using conservative spatial and temporal discretization methods. In this paper, we take a first step and suggest a conservative particle-mesh method for general advection in stream function flow. Similar to the popular vortex-in-cell (VIC) method [4], our method uses Lagrangian particles to describe the advected quantity but the stream function is given on a grid. Following the recently proposed Hamiltonian particle-mesh (HPM) methodology for compressible flows [6,5], we discretise the equations in space to form a Hamiltonian ODE system which

* corresponding author.

Email addresses: colin.cotter@ic.ac.uk (C. J. Cotter), s.reich@ic.ac.uk (S. Reich). 
can then be integrated in time using a symplectic integrator [7]. Hence our particle-mesh method shares the conservative nature of purely particle-based methods such as point vortex and vortex blob methods $[4,8]$ while, at the same time, being as efficiently implementable as traditional particle-mesh methods. The details of the new method are discussed in $\S 2$.

We then apply the method to a weak-wave model [9] in order to demonstrate the benefits of using a conservative method in the context of balance. The weak-wave model used here consists of a slow-moving advection equation coupled to a highly oscillatory linear wave equation. The equations relate to the shallow-water equations in the geostrophic limit. The notion of balance refers to a special region of phase space for which the fast wave variables are essentially slaved to the state of the slow variables. It can be shown [3] that if the Hamiltonian particle-mesh method is used for the weak-wave model then the system will stay close to balance for exponentially long times in the small timescale separation parameter $\epsilon$. Further, we give a technique for setting the exact amount of unbalanced fast motions that will be present in the dynamics. For example it is possible using this technique to specify initial conditions such that the motions are entirely slow - this can be described as 'perfectlybalanced initial conditions'. We give a few numerical results which illustrate the technique.

\section{A Hamiltonian particle-mesh method for advection in stream function flow}

\subsection{Background}

Consider the equation below for the advection of a quantity $q$ by a velocity field $\mathbf{u}(\mathbf{x}, t)$ :

$$
q_{t}+\mathbf{u} \cdot \nabla q=0 \text {. }
$$

If the flow is 2-dimensional and incompressible $(\nabla \cdot \mathbf{u}=0)$, then $\mathbf{u}$ may be written as

$$
\mathbf{u}=\left(-\psi_{y}, \psi_{x}\right)
$$

for some scalar function $\psi$ (called the stream function). Equation (1) may now be expressed in the form

$$
q_{t}=\{q, \psi\},
$$

where the Lie-Poisson bracket $\{\cdot, \cdot\}$ is defined by

$$
\{f, g\}=f_{x} g_{y}-f_{y} g_{x}
$$


Eq. (2) can be viewed as a non-canonical Hamiltonian system with Hamiltonian functional

$$
\mathcal{H}=\int q \psi \mathrm{d}^{2} x
$$

variational derivative

$$
\frac{\delta \mathcal{H}}{\delta q}=\psi
$$

and non-canonical structure operator

$$
\mathcal{J}=\{q, \cdot\}
$$

Hence, eq. (2) may finally be written

$$
\dot{q}=\mathcal{J} \frac{\delta \mathcal{H}}{\delta q} .
$$

This structure applies for the case of:

- the scalar advection equation where the stream function has some predetermined form $\psi=f(\mathbf{x}, t)$,

- the $2 D$ Euler equation where the stream function is obtained from $q$ (which here represents the vorticity) as follows:

$$
\nabla^{2} \psi=q
$$

and

- the quasi-geostrophic equation where the stream function is obtained from $q$ (which here represents the potential vorticity) as follows:

$$
\left(\Delta-f_{0}\right) \psi=q, \quad f_{0} \text { constant. }
$$

For more background, see [8] or [12].

\subsection{Numerical method}

Due to the non-canonical Hamiltonian structure of equation (2), conservative spatial discretizations are not available, in general. This problem can be circumvented by going to the Lagrangian formulation of the advection equation (1). The point vortex and vortex blob method are examples of such an approach (see [8] and [4]). Below we describe a mixed conservative particlemesh discretization that can be easily generalized to the weak-wave models described in $\S 3$. The basic idea follows that of Frank, GotTwald \& REICH [6], FRANK \& REICH [5] for compressible fluids.

The approach of the particle-mesh method is to use a Lagrangian particle discretisation for the $q$-field and to restrict the stream function $\psi$ to a grid. 
First, write $q$ as a weighted sum of 'blobs' centred on $n$ Lagrangian particles moving with the flow:

$$
q(\mathbf{x}, t)=\sum_{i=1}^{n} \alpha_{i} \phi\left(\boldsymbol{X}_{i}(t)-\boldsymbol{x}\right)
$$

where the $\alpha_{i}$ are local constants representing circulation (or vorticity), $\boldsymbol{X}_{i}=$ $\left(X_{i}, Y_{i}\right)^{\top}$ are the positions of the particles (with dynamics to be determined shortly) and $\phi$ is some positive basis function (e.g. a Gaussian) which is scaled to satisfy

$$
\int \phi(\boldsymbol{x}) \mathrm{d}^{2} x=1
$$

Substituting into the Hamiltonian gives

$$
\mathcal{H}(\boldsymbol{Z})=\sum_{i} \alpha_{i} \int \phi\left(\boldsymbol{X}_{i}-\boldsymbol{x}\right) \psi(\boldsymbol{x}) \mathrm{d}^{2} x, \quad \boldsymbol{Z}=\left(\boldsymbol{X}_{1}^{\top}, \boldsymbol{X}_{2}^{\top}, \ldots, \boldsymbol{X}_{n}^{\top}\right)^{\top}
$$

The integral will shortly be approximated over a given grid. For the time being, assume a continuous stream function $\psi$ and exact evaluation of the integral. Next consider the skew symmetric matrix

$$
J_{A}=\left(\begin{array}{cccccc}
0 & \frac{1}{\alpha_{1}} & \ldots & \ldots & 0 & 0 \\
-\frac{1}{\alpha_{1}} & 0 & \ldots & \ldots & 0 & 0 \\
\vdots & \vdots & \ddots & \ddots & \vdots & \vdots \\
0 & 0 & \ldots & \ldots & 0 & \frac{1}{\alpha_{n}} \\
0 & 0 & \ldots & \ldots & -\frac{1}{\alpha_{n}} & 0
\end{array}\right) .
$$

This operator gives rise to the canonical equations of motion

$$
\dot{\boldsymbol{Z}}=J_{A} \nabla_{\boldsymbol{Z}} \mathcal{H}(\boldsymbol{Z})
$$

for the vector $\boldsymbol{Z}$ of Lagrangian particle positions. In terms of the individual particle positions, the following equations of motion are obtained:

$$
\dot{\boldsymbol{X}}_{i}=\nabla_{\boldsymbol{X}_{i}}^{\perp} \int \psi(\boldsymbol{x}) \phi\left(\boldsymbol{X}_{i}-\boldsymbol{x}\right) \mathrm{d}^{2} x
$$

where

$$
\nabla_{\mathbf{x}_{i}}^{\perp}=\left(\frac{\partial}{\partial Y_{i}},-\frac{\partial}{\partial X_{i}}\right)^{\top}
$$

This may be rewritten using integration by parts (for zero or periodic boundary conditions) as

$$
\dot{\boldsymbol{X}}_{i}=-\int \psi(\boldsymbol{x}) \nabla_{\mathbf{x}}^{\perp} \phi\left(\boldsymbol{X}_{i}-\boldsymbol{x}\right) \mathrm{d}^{2} x=\int \phi\left(\boldsymbol{X}_{i}-\boldsymbol{x}\right) \nabla_{\mathbf{x}}^{\perp} \psi(\boldsymbol{x}) \mathrm{d}^{2} x,
$$


where

$$
\nabla_{\mathbf{x}}^{\perp} \psi=\left(\psi_{y},-\psi_{x}\right)^{\top}=\mathbf{u}
$$

and so the velocity of the particle is given as a 'smoothed' average value of the flow velocity around its position, weighted by $\phi$. The velocity of the particle converges to the local fluid velocity as the functions $\phi$ converge to Dirac $\delta$-distributions. All that remains is to approximate the integral in the Hamiltonian as a sum over a grid (which can be done in many different ways). For the example in this paper we use double-periodic boundary conditions and evaluate the integral using the midpoint rule over an $m \times m$ grid with equally spaced grid points $\boldsymbol{x}_{k l}$ so that the Hamiltonian becomes

$$
H=\left(\frac{L}{m}\right)^{2} \sum_{i=1}^{n} \alpha_{i} \sum_{k, l=1}^{m} \phi_{k l}\left(\boldsymbol{X}_{i}\right) \psi_{k l}=\left(\frac{L}{m}\right)^{2} \sum_{i=1}^{n} \alpha_{i} \phi\left(\boldsymbol{X}_{i}\right)^{\top} \boldsymbol{\psi}
$$

where $L$ is the domain length, $\psi_{k l}=\psi\left(\boldsymbol{x}_{k l}\right), \phi_{k l}\left(\boldsymbol{X}_{i}\right)=\phi\left(\boldsymbol{X}_{i}-\boldsymbol{x}_{k l}\right)$, and $\boldsymbol{\psi}$, $\phi$ are corresponding column vectors.

Since the spatial discretization produces a set of Hamiltonian ODEs, a symplectic time discretization should be applied [7, and references therein]. The symplectic integrator used here is the implicit mid-point rule which requires iteration to convergence to remain symplectic. However, for a given stream function $\psi$, the equations of motion decouple into their individual particle contributions so that a Newton-type iteration is very easy to implement.

As a further example, consider the 2D Euler equation, $\psi=\nabla^{-1} q$. The inverse Laplace-operator may be discretised on the grid (using e.g. Fourier transform or finite-differences) to become a matrix $B^{-1}$ and the Hamiltonian becomes

$$
H=\frac{1}{2}\left(\frac{L}{m}\right)^{2} \sum_{i, j} \alpha_{i} \alpha_{j} \phi\left(\boldsymbol{X}_{i}\right)^{\top} B^{-1} \boldsymbol{\phi}\left(\boldsymbol{X}_{j}\right) .
$$

This gives a particle-mesh scheme for the 2D Euler equations. Changing $B^{-1}$ can give a scheme for the quasi-geostrophic equations.

The computational efficiency of this method for discretising the advection equation can be made feasible by using basis functions which are non-zero only for a few grid points surrounding the particle. Some smoothing of the basis functions on the grid may then be necessary to make them into global basis functions. This can also be done in a such a way as to keep the system Hamiltonian by following the approach of [6].

This method combines the conservative structure of the vortex methods with the computational advantages (such as the speed of evaluation of the gradients) of the vortex-in-cell (VIC) method. Another appealing feature of our approach is that it allows for a coupling of Lagrangian advection with dynamic 
processes that are more suitably defined over the fixed Eulerian grid $\boldsymbol{x}_{k l}$. We will demonstrate this below for the weak-wave model.

\section{Weak-wave model}

Nore \& Shepherd [9] proposed a simplified PDE model for the shallow water equations on an $f$-plane (see [12]) very near to geostrophic balance in which a slowly moving incompressible flow is coupled to a quickly oscillating linear wave equation. For simplicity, we discuss a very similar model given below:

$$
q_{t}=\epsilon\{q, h\}, \quad b_{t}=\left(f_{0}-\Delta\right) h+q, \quad h_{t}=-b .
$$

Here $q$ represents the potential vorticity in the shallow-water equations, $b$ represents the velocity divergence $(\nabla . \mathbf{u})$ and $h$ represents the height of the fluid layer. Several nonlinear terms in the shallow-water equations have been dropped so that here the velocity advecting $q$ is incompressible with stream function $\psi=h$. Throughout the paper the constant $f_{0}$ is assumed to be equal to one and $\epsilon$ is a small parameter which represents the timescale separation between the vortical $q$-motion and the motion in the wave modes $b$ and $h$. Note that if $b_{t}=h_{t}=0$ for all times then the quasi-geostrophic model is recovered:

$$
q_{t}=\epsilon\{q, \psi\}, \quad \psi=\left(\Delta-f_{0}\right)^{-1} q .
$$

Note also that if $q=0$ everywhere then the PDE reduces to a linear dispersive wave equation:

$$
b_{t}=\left(f_{0}-\Delta\right) h, \quad h_{t}=-b .
$$

The waves that propagate in this system are called inertia-gravity waves. Of particular interest is the interaction of these waves with areas of non-zero $q$.

This PDE also has a non-canonical Hamiltonian structure. In particular, the structure operator $\mathcal{J}$ is

$$
\mathcal{J}=\left(\begin{array}{ccc}
\epsilon\{q, \cdot\} & 0 & 0 \\
0 & 0 & 1 \\
0 & -1 & 0
\end{array}\right)
$$

and the Hamiltonian is

$$
\mathcal{H}=\mathcal{H}_{\text {advection }}+\mathcal{H}_{\text {wave }}=\int q h \mathrm{~d}^{2} x+\int\left(\frac{b^{2}}{2}+f_{0} \frac{h^{2}}{2}+\frac{|\nabla h|^{2}}{2}\right) \mathrm{d}^{2} x .
$$

Notice that $\mathcal{J}$ is a combination of the standard Lie-Poisson bracket of equation (2) and the canonical symplectic structure in $(b, h)^{\top}$. After writing $\boldsymbol{z}=$ 
$(q, b, h)^{\top}$, the equations may then be written in the abstract form

$$
\boldsymbol{z}_{t}=\mathcal{J} \frac{\delta \mathcal{H}}{\delta \boldsymbol{z}}
$$

The system in the $q$ variable is of the type described in $\S 2$ and so the numerical method of $\S 2.2$ may be used. The main interest for this model is the nature of the exchange of energy between $\mathcal{H}_{\text {wave }}$ and $\mathcal{H}_{\text {advection }}$.

\section{A geometric integrator for the weak wave model}

In this section we generalize the Hamiltonian particle-mesh method of $\S 3$ to the weak-wave model. Once again the method is obtained by first discretising the PDE in space to produce a Hamiltonian ODE, and then using a symplectic method for the time stepping.

In his $\mathrm{PhD}$ thesis [13], Wirosoetisno discretised a very similar weak-wave model using a sine-truncation of the structure operator (see ZEITLIN [14]). However this Hamiltonian truncation is restricted to periodic boundary conditions whereas the method described here is applicable to more general boundary conditions and grids.

To discretise the $q$ equation, use the same method as described in $\S 2.2$ so that

$$
q(\mathbf{x}, t)=\sum_{i=1}^{n} \alpha_{i} \phi\left(\boldsymbol{X}_{i}(t)-\boldsymbol{x}\right)
$$

The integration in the Hamiltonian is discretised on a $m \times m$ double-periodic grid using the midpoint rule and $\nabla h$ is replaced by $D \mathbf{h}$ where the skewsymmetric matrix $D$ approximates the gradient (e.g. using discrete Fourier transforms). The discrete system has structure matrix $J$ given by

$$
J=\left(\begin{array}{ccc}
\epsilon J_{A} & 0 & 0 \\
0 & 0 & \frac{m^{2}}{L^{2}} I \\
0 & -\frac{m^{2}}{L^{2}} I & 0
\end{array}\right),
$$

where $J_{A}$ is the $n \times n$ skew-symmetric matrix (3), and $I$ is the $m \times m$ identity matrix. The Hamiltonian is

$$
H=\left(\frac{L}{m}\right)^{2}\left(\frac{\boldsymbol{b}^{\top} \boldsymbol{b}}{2}+\frac{\boldsymbol{h}^{\top} B \boldsymbol{h}}{2}+\sum_{i=1}^{n} \alpha_{i} \boldsymbol{h}^{\top} \boldsymbol{\phi}\left(\boldsymbol{X}_{i}\right)\right),
$$

where $h_{k l}$ and $b_{k l}$ represent the values of $h$ and $b$ at position $\boldsymbol{x}_{k l}$ on the grid, $\boldsymbol{h}, \boldsymbol{b}$ are associated column vectors, and $B=f_{0} I-L$ ( $I$ is the identity matrix 
and $L=-D^{2}$ ). The time-integration is done via a splitting method. The Hamiltonian is split into $H_{\text {wave }}$ and $H_{\text {advection with }}$

$$
H_{\text {wave }}=\left(\frac{L}{m}\right)^{2}\left(\frac{\boldsymbol{b}^{\top} \boldsymbol{b}}{2}+\frac{\boldsymbol{h}^{\top} B \boldsymbol{h}}{2}\right), \quad H_{\text {advection }}=\left(\frac{L}{m}\right)^{2} \sum_{i=1}^{n} \alpha_{i} \boldsymbol{h}^{\top} \boldsymbol{\phi}\left(\boldsymbol{X}_{i}\right) .
$$

The system for $H_{\text {wave }}$ is

$$
\dot{\boldsymbol{b}}=B \boldsymbol{h}, \quad \dot{\boldsymbol{h}}=-\boldsymbol{b}, \quad \dot{\boldsymbol{X}}_{i}=0,
$$

which is a linear wave equation which may be solved exactly. Write the time- $\Delta t$ flow-map of this system as $\Psi_{1, \Delta t}$. The system for $H_{\text {advection }}$ is

$$
\dot{\boldsymbol{b}}=\boldsymbol{q}(\boldsymbol{X}, \boldsymbol{Y}), \quad \dot{\boldsymbol{h}}=0, \quad \dot{\boldsymbol{X}}_{i}=\epsilon\left(\frac{L}{m}\right)^{2} \nabla_{\boldsymbol{X}_{i}}^{\perp} \phi\left(\boldsymbol{X}_{i}\right)^{\top} \boldsymbol{h} .
$$

This second system may be numerically integrated using the implicit midpoint method (which is symplectic and second-order). The method is

$$
\begin{aligned}
\frac{\boldsymbol{b}^{n+1}-\boldsymbol{b}^{n}}{\Delta t} & =\boldsymbol{q}\left(\boldsymbol{X}^{n+1 / 2}\right), \\
\frac{\boldsymbol{h}^{n+1}-\boldsymbol{h}^{n}}{\Delta t} & =0 \\
\frac{\boldsymbol{X}_{i}^{n+1}-\boldsymbol{X}_{i}^{n}}{\Delta t} & =\epsilon\left(\frac{L}{m}\right)^{2} \nabla_{\boldsymbol{X}_{i}}^{\perp} \boldsymbol{\phi}\left(\boldsymbol{X}_{i}^{n+1 / 2}\right)^{\top} \boldsymbol{h},
\end{aligned}
$$

where

$$
\boldsymbol{X}_{i}^{n+1 / 2}=\frac{\boldsymbol{X}_{i}^{n+1}+\boldsymbol{X}_{i}^{n}}{2}
$$

Note that although this involves the solution of a non-linear system, it decouples into two-by-two systems for each particle, and that after solving these systems the time-stepping for $\boldsymbol{b}$ is explicit. Write the map for this method as $\Psi_{2, \Delta t}$. A second-order symplectic method for the whole geometric integrator can be obtained using the Strang splitting (see e.g. [7]) as shown below:

$$
\Psi_{\Delta t}=\Psi_{1, \Delta t / 2} \circ \Psi_{2, \Delta t} \circ \Psi_{1, \Delta t / 2}
$$

\section{Time-scale separation and normal form results}

The aim of this section is to transform the variables into a form which makes the structure of dynamics more explicit. This transformation is a symplectomorphism (in order to preserve the Hamiltonian structure of the equations) which is obtained from a generating function in a slightly different way from 
usual due to the non-canonical scalings in the structure matrix $J$. Let us first introduce the two vectors

$$
\boldsymbol{X}=\left(X_{1}, X_{2}, \ldots, X_{n}\right)^{\top}, \quad \boldsymbol{Y}=\left(Y_{1}, Y_{2}, \ldots, Y_{n}\right)^{\top}
$$

of all the $x$ - and $y$-coordinates of the Lagrangian particles. We also define the vector of $q$-values over the grid given by

$$
\boldsymbol{q}(\boldsymbol{X}, \boldsymbol{Y})=\sum_{i=1}^{n} \alpha_{i} \phi\left(\boldsymbol{X}_{i}\right)
$$

The chosen generating function is

$$
S=\left(\frac{L}{m}\right)^{2} \overline{\boldsymbol{b}}^{\top}\left(\boldsymbol{h}+B^{-1} \boldsymbol{q}(\overline{\boldsymbol{X}}, \boldsymbol{Y})\right)+\frac{1}{\epsilon} \sum_{i=1}^{n} \alpha_{i} \bar{X}_{i} Y_{i}
$$

The new canonical variables $(\overline{\boldsymbol{b}}, \overline{\boldsymbol{h}}, \overline{\boldsymbol{X}}, \overline{\boldsymbol{Y}})$ are calculated as follows:

$$
\begin{aligned}
\boldsymbol{b} & =\left(\frac{m}{L}\right)^{2} \frac{\partial S}{\partial \boldsymbol{h}}=\overline{\boldsymbol{b}} \\
\overline{\boldsymbol{h}} & =\left(\frac{m}{L}\right)^{2} \frac{\partial S}{\partial \overline{\boldsymbol{b}}}=\boldsymbol{h}+B^{-1} \boldsymbol{q}(\overline{\boldsymbol{X}}, \boldsymbol{Y})
\end{aligned}
$$

and

$$
\begin{aligned}
& X_{i}=\epsilon \alpha_{i}^{-1} \frac{\partial S}{\partial Y_{i}}=\bar{X}_{i}+\epsilon \alpha_{i}^{-1}\left(\frac{L}{m}\right)^{2} \frac{\partial}{\partial Y_{i}} \bar{b}^{\top} B^{-1} \boldsymbol{q}(\overline{\boldsymbol{X}}, \boldsymbol{Y}), \\
& \bar{Y}_{i}=\epsilon \alpha_{i} \frac{\partial S}{\partial \bar{X}_{i}}=Y_{i}+\epsilon \alpha_{i}\left(\frac{L}{m}\right)^{2} \frac{\partial}{\partial \bar{X}_{i}} \overline{\boldsymbol{b}}^{\top} B^{-1} \boldsymbol{q}(\overline{\boldsymbol{X}}, \boldsymbol{Y})
\end{aligned}
$$

for $i=1, \ldots, n$. Note that $\overline{\boldsymbol{X}}$ is defined implicitly in the above equations.

This transformation is a symplectomorphism so the equations in the new variables may be obtained simply by substituting them into the Hamiltonian. This gives 


$$
\begin{aligned}
& H=\left(\frac{L}{m}\right)^{2}\left(\frac{\boldsymbol{b}^{\top} \boldsymbol{b}}{2}+\frac{\boldsymbol{h}^{\top} B \boldsymbol{h}}{2}+\boldsymbol{h}^{\top} \boldsymbol{q}(\boldsymbol{X}, \boldsymbol{Y})\right) \\
& =\left(\frac{L}{m}\right)^{2}\left(\frac{\bar{b}^{\top} \bar{b}}{2}+\frac{\left(\overline{\boldsymbol{h}}-B^{-1} \boldsymbol{q}(\overline{\boldsymbol{X}}, \boldsymbol{Y})\right)^{\top} B\left(\overline{\boldsymbol{h}}-B^{-1} \boldsymbol{q}(\overline{\boldsymbol{X}}, \boldsymbol{Y})\right)}{2}\right. \\
& \left.+\left(\overline{\boldsymbol{h}}-B^{-1} \boldsymbol{q}(\overline{\boldsymbol{X}}, \boldsymbol{Y})\right)^{\top} \boldsymbol{q}(\boldsymbol{X}, \boldsymbol{Y})\right) \\
& =\left(\frac{L}{m}\right)^{2}\left(\frac{\overline{\boldsymbol{b}}^{\top} \overline{\boldsymbol{b}}}{2}+\frac{\overline{\boldsymbol{h}}^{\top} B \overline{\boldsymbol{h}}}{2}-\overline{\boldsymbol{h}}^{\top} \boldsymbol{q}(\overline{\boldsymbol{X}}, \boldsymbol{Y})+\frac{\boldsymbol{q}(\overline{\boldsymbol{X}}, \boldsymbol{Y})^{\top} B^{-1} \boldsymbol{q}(\overline{\boldsymbol{X}}, \boldsymbol{Y})}{2}\right. \\
& \left.+\overline{\boldsymbol{h}}^{\top} \boldsymbol{q}(\boldsymbol{X}, \boldsymbol{Y})-\boldsymbol{q}(\overline{\boldsymbol{X}}, \boldsymbol{Y})^{\top} B^{-1} \boldsymbol{q}(\boldsymbol{X}, \boldsymbol{Y})\right) \\
& =\left(\frac{L}{m}\right)^{2}\left(\frac{\overline{\boldsymbol{b}}^{\top} \overline{\boldsymbol{b}}}{2}+\frac{\overline{\boldsymbol{h}}^{\top} B \overline{\boldsymbol{h}}}{2}-\frac{\boldsymbol{q}(\overline{\boldsymbol{X}}, \overline{\boldsymbol{Y}})^{\top} B^{-1} \boldsymbol{q}(\overline{\boldsymbol{X}}, \overline{\boldsymbol{Y}})}{2}+\epsilon S_{\epsilon}(\overline{\boldsymbol{h}}, \overline{\boldsymbol{b}}, \overline{\boldsymbol{X}}, \overline{\boldsymbol{Y}})\right) .
\end{aligned}
$$

To obtain the last equality we made use of

$$
\boldsymbol{q}(\overline{\boldsymbol{X}}, \overline{\boldsymbol{Y}})=\boldsymbol{q}(\overline{\boldsymbol{X}}, \boldsymbol{Y})+\mathcal{O}(\epsilon)=\boldsymbol{q}(\boldsymbol{X}, \boldsymbol{Y})+\mathcal{O}(\epsilon)
$$

and collected all terms of size $\mathcal{O}(\epsilon)$ and smaller into a function $S_{\epsilon}$.

In these new coordinates the Hamiltonian consists of a fast wave part

$$
\bar{H}_{\text {wave }}=\left(\frac{L}{m}\right)^{2}\left(\frac{\overline{\boldsymbol{b}}^{\top} \overline{\boldsymbol{b}}}{2}+\frac{\overline{\boldsymbol{h}}^{\top} B \overline{\boldsymbol{h}}}{2}\right),
$$

a slow particle motion part

$$
\bar{H}_{\text {advection }}=-\left(\frac{L}{m}\right)^{2} \frac{\boldsymbol{q}(\overline{\boldsymbol{X}}, \overline{\boldsymbol{Y}})^{\top} B^{-1} \boldsymbol{q}(\overline{\boldsymbol{X}}, \overline{\boldsymbol{Y}})}{2}
$$

and a small $\mathcal{O}(\epsilon)$ perturbation. If this perturbation is zero, then the Hamiltonian represents uncoupled separated fast wave and slow particle motion. The effect of the coupling term on the slow and fast dynamics is of much interest. It can be shown that for analytic basis functions there exists a second symplectic change of coordinates

$$
\Phi(\overline{\boldsymbol{b}}, \overline{\boldsymbol{h}}, \overline{\boldsymbol{X}}, \overline{\boldsymbol{Y}})=(\tilde{\boldsymbol{b}}, \tilde{\boldsymbol{h}}, \tilde{\boldsymbol{X}}, \tilde{\boldsymbol{Y}})
$$

for which the fast wave energy

$$
\tilde{H}_{\text {wave }}=\left(\frac{L}{m}\right)^{2}\left(\frac{\tilde{\boldsymbol{b}}^{\top} \tilde{\boldsymbol{b}}}{2}+\frac{\tilde{\boldsymbol{h}}^{\top} B \tilde{\boldsymbol{h}}}{2}\right)
$$


is conserved for times which are exponentially long in $\epsilon^{1 / n}$ if the wave energy is sufficiently small ( $n$ is the number of wave frequencies present).

This result is based on the method of PöscheL [10] and the references therein, and is described in detail for this particular model in CoTTER [3]. One would wish to extend this result to the case for which $h$ and $b$ are now continuous wave fields but there are still a finite number of particles (called the waveblob model). The is not possible in general because $\epsilon^{1 / n} \rightarrow 1$ as $n \rightarrow \infty$ which means that the exponential bound is no longer small. However it is possible to obtain a good exponential estimate for a related 1D problem if when $h$ and $b$ are initialised with analytic functions which are sufficiently smooth [3]. The result is based on a method developed by BAMBUsi [1] for the case of semi-linear wave equations. This extended result indicates that this property of the semi-discretised scheme is reflecting important behaviour of the PDE it is approximating.

To interpret this result in terms of the dynamics consider the inverse image under $\Phi$ of the phase surface

$$
\{(\tilde{\boldsymbol{b}}, \tilde{\boldsymbol{h}}, \tilde{\boldsymbol{X}}, \tilde{\boldsymbol{Y}}): \tilde{\boldsymbol{h}}=0, \tilde{\boldsymbol{b}}=0\}
$$

This inverse image in the untransformed coordinates is called the slow manifold which is invariant for exponentially long times. On the slow manifold the dynamics of $\boldsymbol{h}$ and $\boldsymbol{b}$ are determined entirely by the slow variables. More generally consider the family of tori given by the inverse image of the phase surface:

$$
\mathcal{M}_{r}=\left\{(\tilde{\boldsymbol{b}}, \tilde{\boldsymbol{h}}, \tilde{\boldsymbol{X}}, \tilde{\boldsymbol{Y}}):\left(\frac{L}{m}\right)^{2}\left(\frac{\tilde{\boldsymbol{b}}^{\top} \tilde{\boldsymbol{b}}}{2}+\frac{\tilde{\boldsymbol{h}}^{\top} B \tilde{\boldsymbol{h}}}{2}\right)=r\right\} .
$$

For small enough $r$, these tori are very 'sticky'; i.e. if the system is initialised on such a torus then the evolution will remain exponentially close to the torus for exponentially long times. The dynamics now looks like fast small-amplitude nonlinear oscillations about a slow mean trajectory in phase space.

\section{Application of result to numerical integration}

The normal form results also extend to the fully discretised model. As the time-integrator is symplectic, it represents the solution of the problem with a modified Hamiltonian which differs by $\mathcal{O}\left(\Delta t^{2}\right)$ from the original Hamiltonian. This modified Hamiltonian also has a slow manifold which converges to the original slow manifold as $\Delta t$ converges to 0 . This means that the long time exchange of energy between the slow and fast variables has the same properties 
as the semi-discretised system (see e.g. REICH [11]). The modified equations also have 'sticky' tori centered on the slow manifold which converge to the tori in the original system. If a non-symplectic time integrator were used then the wave energy in the transformed variables would either decay or grow in time and the numerical method would not have the correct long-time properties; i.e. the tori will no longer be 'sticky'.

A useful application can be made from this result. Consider the time-dependent Hamiltonian

$$
H=\left(\frac{L}{m}\right)^{2}\left(\frac{\boldsymbol{b}^{\top} \boldsymbol{b}}{2}+\frac{\boldsymbol{h}^{\top} B \boldsymbol{h}}{2}+f(\epsilon t) \boldsymbol{h}^{\top} \boldsymbol{q}(\boldsymbol{X}, \boldsymbol{Y})\right),
$$

where $f$ is a smooth function which satisfies

$$
f \rightarrow 0 \text { as } t \rightarrow-\infty, \quad f \rightarrow 1 \text { as } t \rightarrow \infty,
$$

such as

$$
f(t)=\frac{1}{2}(1+\tanh (t)) .
$$

This means that for large negative times, the system is just a set of uncoupled wave equations, and for large positive times we recover the particle-mesh weakwave model. The idea here is to gradually increase the coupling up from zero to keep the $b$ and $h$ fields balanced. This is a method which is often used on an ad hoc basis, but the discussion which follows shows that here this embedding technique has a rigorous foundation.

This system can be reinterpreted as a time-dependent Hamiltonian system by introducing conjugate variables $s$ and $e$ which vary on the slow timescale; i.e.

$$
\left(\begin{array}{c}
\dot{e} \\
\dot{s}
\end{array}\right)=\left(\begin{array}{cc}
0 & \epsilon \\
-\epsilon & 0
\end{array}\right)\left(\begin{array}{c}
\frac{\partial H}{\partial e} \\
\frac{\partial H}{\partial s}
\end{array}\right) \text {, }
$$

and by introducing the extended Hamiltonian:

$$
H=\left(\frac{L}{m}\right)^{2}\left(\frac{\boldsymbol{b}^{\top} \boldsymbol{b}}{2}+\frac{\boldsymbol{h}^{\top} B \boldsymbol{h}}{2}+f(s) \boldsymbol{h}^{\top} \boldsymbol{q}(\boldsymbol{X}, \boldsymbol{Y})\right)-e .
$$

Note that $s=\epsilon t$ if $s\left(t_{0}\right)=\epsilon t_{0}$ for some initial value $t_{0}$. For this problem we introduce a change of variables by the extended generating function

$$
\bar{S}=\left(\frac{L}{m}\right)^{2} \overline{\boldsymbol{b}}^{\top}\left(\boldsymbol{h}+f(s) B^{-1} \boldsymbol{q}(\overline{\boldsymbol{X}}, \boldsymbol{Y})\right)+\frac{1}{\epsilon} \sum_{i=1}^{n} \alpha_{i} \bar{X}_{i} Y_{i}+\frac{1}{\epsilon} \bar{e} s .
$$

In a similar way to before, this brings about a change of coordinates given below: 


$$
\begin{aligned}
& \boldsymbol{b}=\left(\frac{m}{L}\right)^{2} \frac{\partial S}{\partial \boldsymbol{h}}=\overline{\boldsymbol{b}} \\
& \overline{\boldsymbol{h}}=\left(\frac{m}{L}\right)^{2} \frac{\partial S}{\partial \overline{\boldsymbol{b}}}=\boldsymbol{h}+f(s) B^{-1} \boldsymbol{q}(\overline{\boldsymbol{X}}, \boldsymbol{Y}),
\end{aligned}
$$

and

$$
\begin{aligned}
& X_{i}=\epsilon \alpha_{i}^{-1} \frac{\partial S}{\partial Y_{i}}=\bar{X}_{i}+\epsilon \alpha_{i}^{-1} f(s)\left(\frac{L}{m}\right)^{2} \frac{\partial}{\partial Y_{i}} \bar{b}^{\top} B^{-1} \boldsymbol{q}(\overline{\boldsymbol{X}}, \boldsymbol{Y}) \\
& \bar{Y}_{i}=\epsilon \alpha_{i}^{-1} \frac{\partial S}{\partial \bar{X}_{i}}=Y_{i}+\epsilon \alpha_{i}^{-1} f(s)\left(\frac{L}{m}\right)^{2} \frac{\partial}{\partial \bar{X}_{i}} \overline{\boldsymbol{b}}^{\top} B^{-1} \boldsymbol{q}(\overline{\boldsymbol{X}}, \boldsymbol{Y})
\end{aligned}
$$

for $i=1, \ldots, n$, together with

$$
\begin{aligned}
& e=\epsilon \frac{\partial S}{\partial s}=\bar{e}+\epsilon f^{\prime}(s) B^{-1} \boldsymbol{q}(\overline{\boldsymbol{X}}, \boldsymbol{Y}), \\
& \bar{s}=\epsilon \frac{\partial S}{\partial \bar{e}}=s .
\end{aligned}
$$

This coordinate change transforms the Hamiltonian to the form

$$
\left(\frac{L}{m}\right)^{2}\left(\frac{\overline{\boldsymbol{b}}^{\top} \overline{\boldsymbol{b}}}{2}+\frac{\overline{\boldsymbol{h}}^{\top} B \overline{\boldsymbol{h}}}{2}-\frac{\boldsymbol{q}(\overline{\boldsymbol{X}}, \overline{\boldsymbol{Y}})^{\top} B^{-1} \boldsymbol{q}(\overline{\boldsymbol{X}}, \overline{\boldsymbol{Y}})}{2}+\epsilon R_{\epsilon}(\overline{\boldsymbol{h}}, \overline{\boldsymbol{b}}, \overline{\boldsymbol{X}}, \overline{\boldsymbol{Y}} e, s)\right)-\bar{e}
$$

This is now in a very similar form to before, and the result of the previous section extends to this system; i.e. there exists a change of coordinates

$$
\bar{\Phi}(\overline{\boldsymbol{h}}, \overline{\boldsymbol{b}}, \overline{\boldsymbol{X}}, \overline{\boldsymbol{Y}}, \bar{e}, \bar{s})=(\tilde{\boldsymbol{h}}, \tilde{\boldsymbol{b}}, \tilde{\boldsymbol{X}}, \tilde{\boldsymbol{Y}}, \tilde{e}, \tilde{s})
$$

for which the fast wave energy

$$
\tilde{H}_{\text {wave }}=\left(\frac{L}{m}\right)^{2}\left(\frac{\tilde{\boldsymbol{b}}^{\top} \tilde{\boldsymbol{b}}}{2}+\frac{\tilde{\boldsymbol{h}}^{\top} B \tilde{\boldsymbol{h}}}{2}\right)
$$

is again conserved for exponentially long times. Note that that for early times when $f(s)=0$, the Hamiltonian satisfies $H=H_{\text {wave }}$ and so the transformation due to $\bar{S}$ and the second transformation $\bar{\Phi}$ are both equal to the identity (because $H_{\text {wave }}$ is already conserved in the original variables). Note also that for late times with $f(s)=1$, the system reverts to the original weak-wave system described in this paper, and the transformation due to $\bar{S}$ is the same transformation described in $\S 5$. This means that by using this embedding technique it is possible to obtain initial conditions for the weak-wave model where the exact amount of conserved wave energy in the transformed coordinates is known, even though it is not possible to calculate the value of these coordinates exactly. In particular, if the wave energy is initialised at zero for 